\title{
Delineation of molecular interactions of plant growth promoting bacteria induced $\beta$-1,3-glucanases and guanosine triphosphate ligand for antifungal response in rice: a molecular dynamics approach
}

\author{
Yachana Jha ${ }^{1,9} \cdot$ Budheswar Dehury $^{2}$. S. P. Jeevan Kumar ${ }^{3}$. Anurag Chaurasia ${ }^{4}$. Udai B. Singh ${ }^{5}$. \\ Manoj Kumar Yadav ${ }^{6}$ U. B. Angadi ${ }^{10} \cdot$ Rajiv Ranjan $^{7} \cdot$ Minaketan Tripathy $^{8} \cdot$ R. B. Subramanian ${ }^{9} \cdot$ Sunil Kumar $^{5,10}$. \\ Jesus Simal-Gandara ${ }^{11}$ (D)
}

Received: 14 October 2021 / Accepted: 7 December 2021 / Published online: 16 December 2021

(c) The Author(s) 2021

\begin{abstract}
Background The plant growth is influenced by multiple interactions with biotic (microbial) and abiotic components in their surroundings. These microbial interactions have both positive and negative effects on plant. Plant growth promoting bacterial (PGPR) interaction could result in positive growth under normal as well as in stress conditions.

Methods Here, we have screened two PGPR's and determined their potential in induction of specific gene in host plant to overcome the adverse effect of biotic stress caused by Magnaporthe grisea, a fungal pathogen that cause blast in rice. We demonstrated the glucanase protein mode of action by performing comparative modeling and molecular docking of guanosine triphosphate (GTP) ligand with the protein. Besides, molecular dynamic simulations have been performed to understand the behavior of the glucanase-GTP complex.

Results The results clearly showed that selected PGPR was better able to induce modification in host plant at morphological, biochemical, physiological and molecular level by activating the expression of $\beta-1,3$-glucanases gene in infected host plant. The docking results indicated that Tyr75, Arg256, Gly258, and Ser223 of glucanase formed four crucial hydrogen bonds with the GTP, while, only Val220 found to form hydrophobic contact with ligand.

Conclusions The PGPR able to induce $\beta$-1,3-glucanases gene in host plant upon pathogenic interaction and $\beta$-1,3-glucanases form complex with GTP by hydrophilic interaction for induction of defense cascade for acquiring resistance against Magnaporthe grisea.
\end{abstract}

\section{Graphical abstract}

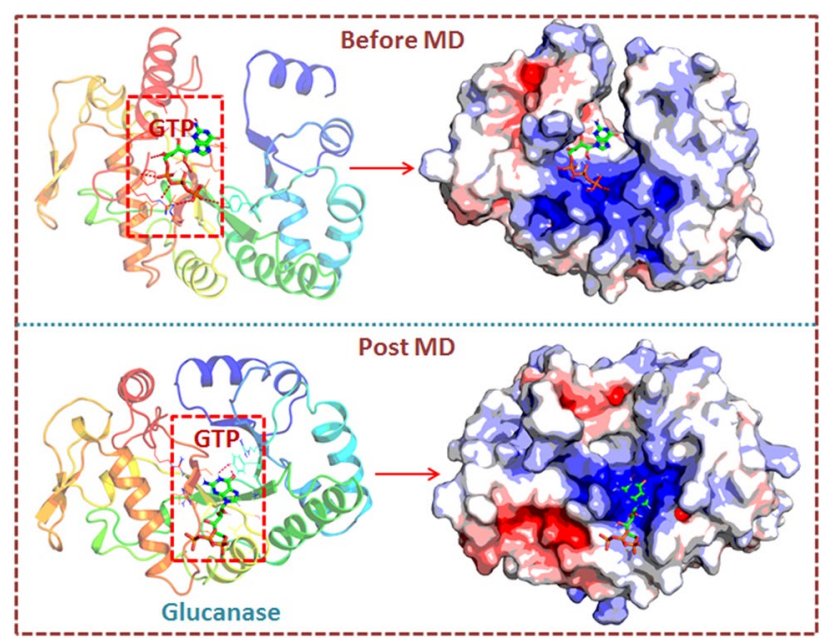

Extended author information available on the last page of the article 
Keywords Biotic stress · Dot blot analysis · $\beta$-1,3-glucanases · GTP ligand · Molecular dynamics simulation

\section{Introduction}

Different types of soil microbes continuously interact with roots of plant in search of food, but it does not result in plant disease frequently, which implies that the plants develop an efficient mechanism to discriminate between normal and potential pathogens. Efficient defense mechanism is induced in plants to check the colonization of root by surrounding phytopathogens [1]. Upon sensing pathogens in their vicinity, a series of defense activities are activated in plant, like stimulation of antimicrobial compounds, closure of stomata, accumulation of lytic enzymes, cell wall lignification, rapid oxidative burst and production of multi-level defense signals that work in specific coordination to block the pathogens interaction with the plant cell due to which plant occasionally get infected by pathogens [2-4].

Plants respond to the pathogens by varying degrees of defense responses. During plant infection, the penetration of pathogens usually rely on the precise time-course of the pathogen discernment by the host plant cells and initiate various biochemical reaction resulting in production of reactive oxygen species (ROS), secondary metabolites, and pathogenesis-related proteins (PRs) and all employed in combination to activate proper defense mechanism against the pathogen infection [5-7]. Synthesis of pathogenesisrelated (PR') proteins like $\beta-1,3$ glucanases, chitinases, peroxidases, hydroxyl proline-rich glycoproteins, phenylpropanoid and flavonoid directly or indirectly play an important role in the process of pathogenesis. These $\beta$-1,3-glucanases (EC 3.2.1.39) present in plants play key role in trafficking of materials across the cell membrane, cell division as well as in defense against pathogen. $\beta$-1,3-glucanases play important roles in fighting against the pathogenic fungus by PR-2 family proteins in plants $[8,9]$. $\beta$-1,3-glucanases catalyze the breakdown of 1,3-D-glucosidic linkages in $\beta$-1,3-D-glucan, which is the main cell wall component in fungi.

The plant roots remain intensely colonized by different types of soil microorganisms like bacteria and fungi, as root exudates represent a rich source of nutrients for their growth and establishment. This group of bacteria is also known as plant growth promoting rhizobacteria (PGPR), which play an effective role in plant growth promotion as well as in alleviating the adverse effects of various biotic and abiotic stresses and are involved in many metabolic and physiological processes of plant [10]. Such microorganisms form an association with the plant, and in turn protect the host plant from adverse environmental stresses by producing or regulating the bioactive compounds such as $\beta$-1,3-glucanases (lytic enzymes) and antibiotics.
These microbial bioactive compounds have imperative responsibilities in the plant development and growth by participating in various mechanisms like providing resistance against biotic and abiotic stress factors, protecting plants from numerous phytopathogens and improving the nutrient acquisition ability, and inducing the plant development and yield under stress conditions [11]. The PGPR are non-pathogenic soil bacteria, which could be employed as a sustainable alternative to manage and control the phytopathogens than harmful chemical pesticides.

The PGPR also produce wide range of PR proteins, which coordinate in developmental control and induce defense response against pathogens in the host plant. Interaction of PGPR with the host plant result in induction of induced systemic resistance (ISR). The PGPR mediated ISR are different from pathogen-induced systemic acquired resistance (SAR) in many aspects at molecular level. While pathogen induced SAR is similar to PGPR mediated ISR in developing resistance towards a broad variety of phytopathogens and spread the resistance to uninfected plant parts [12]. The aim of the present study was to explore the potential of PGPR in the induction of $\beta$-1,3- glucanase gene expression after fungal interaction in paddy crop. Besides, molecular modeling of glucanase and its interaction study with GTP ligand in silico was performed.

The interactions of glucanase with GTP can be explored through the molecular dynamics simulation (MD) studies. Therefore, in the present study we employed the molecular modeling technique along with MD simulations to infer the probable mode of binding of glucanase to GTP. The principal component analysis (PCA), ensemble clustering and molecular mechanics-based binding free energy (MM/ PBSA) calculations were employed to elucidate the global motions and the energetic components which drive the interaction of glucanase with GTP. To the best of our knowledge, this is the first such report on fungal pathogen infected paddy crop, where in-silico study of $\beta$-1,3-glucanases interaction with GTP ligand has been complemented with the wet lab experiments.

\section{Materials and methods}

\section{Isolation, identification and inoculation of PGPR}

In this study, two strains of Pseudomonas pseudoalcaligenes and Bacillus pumilus were isolated from the paddy rhizosphere as per the procedure illustrated by Jha et al. [13]. Among them, the isolated strains were identified by PCR amplification of $16 \mathrm{~S}$ rDNA using $16 \mathrm{~S}$ universal primer $8 \mathrm{~F}$ : 
5'AGAG TTTGATCCTGGCTCAG3' and 1510R: 5'GGC TACCTTG TTACGTA3'. The amplified products were sequenced, analyzed by BLAST and submitted to the NCBI database with accession numbers EU921258 and EU921259, respectively. The seeds of rice variety GJ-17 were procured from main rice research center, Navagam, Anand, Gujarat. Aseptic rice seedlings (four days age) of both single and mixture were transferred to culture vials having Hoagland's nutrient medium $(400 \mu \mathrm{L})$ coupled with $400 \mu \mathrm{L}$ of micronutrients and agar $1 \%(\mathrm{w} / \mathrm{v})$ in $40 \mathrm{~mL}$ distilled water. Before transfer, B. pumilus and $P$. pseudoalcaligenes bacteria were added to the medium $\left(6 \times 10^{8} \mathrm{cfu} \mathrm{mL}^{-1}\right)$ and incubated at $27^{\circ} \mathrm{C}$ in a $12 \mathrm{~h}$ light-dark cycle in a growth chamber.

\section{RNA extraction and RT-PCR from isolated RNA}

One gram of fresh paddy leaves from all the treatments (eight samples -four controls, plant inoculated with Bacillus sp, plant inoculated with Pseudomonas sp. and plant inoculated with both in absence of pathogen similarly four others in presence of pathogen) were used for the extraction of RNA. Extraction of RNA was performed in complete nuclease free condition using diethyl pyrocarbonate (DEPC) as per the protocol [14]. The obtained RNA was quantified by nano-drop spectrophotometer, determined quality and stored at $-20{ }^{\circ} \mathrm{C}$ for further use.

RT-PCR of the extracted RNA was performed with the help of RT- PCR Kit (Bangalore Genei) instructions. Two degenerate primers for $\beta$-1,3-glucanase with random oligo dT primers were used for the amplification of $\beta-1,3-$ glucanase genes. The gene specific primers reverse 5'-GGT TCTCGTTGAACATGGCGA-3'and forward 5'-GTGTCT GCTATGGCGTTGTCG-3'; were designed and synthesized by Invitrogen Ltd. The reactions were carried out in duplicate for the different primer combinations. PCR amplification of cDNA was carried out in the next step of RT-PCR reaction with $2 \mu \mathrm{L}$ each of forward and reverse primers, 1.25 $\mu \mathrm{L}$ of $10 \times$ assay buffer, $1 \mu \mathrm{L}$ dNTP mix, $0.25 \mu \mathrm{L}$ Taq DNA polymerase, $3 \mu \mathrm{L} \mathrm{cDNA}$ and $3 \mu \mathrm{L}$ nuclease free water. The PCR conditions were: initial denaturation at $94{ }^{\circ} \mathrm{C}$ for $2 \mathrm{~min}$, denaturation $94{ }^{\circ} \mathrm{C}$ for $1 \mathrm{~min}$, annealing of primer for $1 \mathrm{~min}$, extension at $72{ }^{\circ} \mathrm{C}$ for $4 \mathrm{~min}$ and $72{ }^{\circ} \mathrm{C}$ for $10 \mathrm{~min}$ for 30 cycles.

\section{DNA elution from the gel}

DNA band of interest was excised from the agarose gel using a sharp sterile blade. Gel piece was weighed and larger piece was crushed into smaller pieces. $2.5 \mathrm{~mL}$ of sodium iodide solution was added and the gel was incubated in a water bath at $50-55{ }^{\circ} \mathrm{C}$ for 2 to $3 \mathrm{~min}$ to solubilize it. To the solubilized gel, $15 \mu \mathrm{L}$ of glass solution was added to the sample containing $5 \mu \mathrm{L}$ of DNA. The contents were mixed thoroughly and spun at $12,000 \mathrm{~g}$ for $30 \mathrm{~s}$. Supernatant was discarded and the pellet containing DNA was taken and wash buffer was added to remove the glass solution, vortexed and spun at $12,000 \mathrm{~g}$ for $30 \mathrm{~s}$ and again the supernatant was discarded. The tubes were incubated at $37{ }^{\circ} \mathrm{C}$ in the water bath for 10 min. For elution of DNA, the pellet was re-suspended in $1 \mathrm{X}$ TE buffer, vortexed and incubated at $45^{\circ} \mathrm{C}$ for $5 \mathrm{~min}$. This step was repeated thrice, all the fractions were pooled and finally centrifuged at $5000 \mathrm{~g}$ for $2 \mathrm{~min}$ to remove any traces of glass solution. Efficiency of elution was checked on $1.5 \%(\mathrm{w} / \mathrm{v})$ agarose gel. The eluted gel band was sequenced by Xplorigen Technologies and obtained sequence was analyzed for multiple sequence alignment by Clustal W software. The ExPASy translate tool was used to translate the obtained nucleotide sequences into amino acid sequences. The longest open reading frame was selected for protein BLAST search against non-redundant database of NCBI to search for similar proteins.

\section{RNA dot blot assay}

Fluorescence-labeled probes for RNA dot blot assay were designed in the lab and obtained from Genei, Bangalore, India. The purified $6 \mu \mathrm{g}$ of RNA was directly applied on the nylon membrane by automatic dispenser for RNA dot blot assay and was vacuum baked for $2 \mathrm{~h}$ at $80^{\circ} \mathrm{C}$ for fixation of RNA on the membrane. The membrane was treated with prehybridization buffer to block the free sites on the membrane to prevent unspecific binding of probe. Then biotin labeled gene specific probe was used for hybridization at optimum temperature and salt concentrations. The hybridized membrane was washed 2-3 times to remove unbounded probe and used for detection. Diluted HRP- streptavidin conjugate was used for detection at room temperature in the presence of substrate solution with gentle swirling until a blue colour band developed. The detection reaction was according to the manufacturer's instruction (Bangalore Genei). The spot density of the RNA dot blot was measured by Alpha Ease FC Imaging software (Alpha Innotech).

\section{Molecular modeling of glucanase and docking studies with GTP}

The amino acid sequence of beta-1,3-glucanase (Oryza sativa indica) was extracted from NCBI database (O. sativa; accession no. ADO34999). Templates for the comparative modeling were identified using the BLASTp search against the Brook Haven Protein Data Bank (PDB) with default parameters [15-18]. The sequence alignments of the target and template were carried out using Multalin program [19]. The 3-dimensional model of beta-1,3-glucanase was generated using the program MODELLER version $9.21[20,21]$. 
Roughly 200 models were generated by the modeller based on the lowest value of the probability density function and the fewest restraint violations. Out of the generated models were used for further downstream analysis. The energy minimization was performed on the selected model. We first conducted energy minimization with side chains and then subsequently applied it to the main chain of a $\mathrm{C} \alpha$ backbone. All the calculations were performed using ACCELRYS Discovery Studio 2.5 (Accelrys Inc. San Diego, CA 92,121, USA) software suites. STRIDE was used for the prediction of secondary structure of the modelled glucanase protein. The modelled structure was then superimposed on the template without altering the coordinate systems of atomic position in the template. The residue profiles of the three-dimensional models were further checked using VERIFY3D (http://www.doe-mbi.ucla.edu/Services/Verify3D. $\mathrm{html}$ ). PROCHECK analysis was performed to assess the stereo chemical properties of the three-dimensional models and Ramachandran plots [22].

The homology modelled structure of glucanase was used for docking with the ligand GTP. For docking analysis, we downloaded the structure of GTP from the PubChem database (http://pubchem.ncbi.nlm.nih.gov). An attempt was made to understand the binding of GTP on glucanase interface AutoDock version 4.2 [23] and AutoDocktools 1.5.6.were applied. Prior to this, protein were prepared and added polar-hydrogen atoms using Gasteiger charges calculation. Ligand centered map was developed having 0.375 $\AA$ spacing with grid dimensions of $46 \times 46 \times 46 \AA^{3}$ (x-y-z) encompassing the binding interfaces of the protein. While performing docking, default settings were applied with GA run to 300 . The best docked conformation was selected by MD optimization considering the parameters such as higher hydrogen-bonds, least free energy of binding and interatomic-bonding patterns. BIOVIA Discovery Studio Visualizer version 4.5 and PyMOL were deployed to elucidate the inter-molecular contacts between GTP with glucanase.

\section{Molecular dynamics simulation of glucanase-GTP complex}

MD simulations of glucanase-GTP complex were done to explore the stability, conformational flexibility and dynamic behavior of all-atoms [24, 25]. Ligand topology was deduced from CHARMM general force field (https://cgenff.umary land.edu/) [26] and force fields of protein building was done using GROMACSv2019.4 package [27]. The structure was solvated in a cubic water box in TIP3P water model. The solvated system was then electro-neutralized by addition of $0.15 \mathrm{M} \mathrm{NaCl}$. Energy minimization was performed to remove bad contacts in the complex system using a steepest descent algorithm in 5000 steps. Equilibration was conducted through NVT and NPT ensembles for $10 \mathrm{~ns}$ at $300 \mathrm{~K}$.
The non-hydrogen atoms of the ligand were restrained. Using leapfrog integrator, production of MD was carried out for $50 \mathrm{~ns}$. GROMACS utility toolkits were employed to understand the structural dynamics and intrinsic stability of the Glucanase-complex system. Using the snapshots from MD trajectory, a number of dynamics stability parameters were computed to understand the structural-dynamics of the MD system which includes root mean square deviation (RMSD), C $\alpha$-root mean squared fluctuations (RMSF) and intermolecular hydrogen bond (H-bond) distributions. XMGrace was employed to generate the 2D graphs and images were rendered using BIOVIA DSV and PyMOL.

\section{Results}

As PGPR's are supposed to develop resistance against pathogen, which remain coupled with the corresponding induction in plant gene expression. Total RNA was isolated from the PGPR inoculated paddy leaves after one week of fungal infection and used for the synthesis of cDNA by reverse transcriptase enzyme. The obtained product was used for subsequent amplification of $\beta$-1,3-glucanases gene by PCR using the gene specific primers (Fig. S1). Induction of $\beta-1,3$ glucanase gene in PGPR inoculated plant after pathogen infection was characterized by RT-PCR analysis. The obtained sequences were gathered to establish a consensus sequence. It was uniformly inducted by the inoculation of paddy with either single isolate or in combination. The $\beta$-1,3-glucanases gene length of $1.05 \mathrm{~kb}$ DNA was assembled and submitted to NCBI data base having accession no HM569719.1.

\section{RNA dot blot assay}

The differential changes in mRNA level of PGPR inoculated plant after specific stress treatment was analyzed by RNA-dot blot assay. The differential expression of $\beta-1,3-$ glucanases gene at the level of transcription under each set of condition was confirmed by RNA dot blot using a eukaryotic translation factor eTEF1 $\alpha$ as an internal standard. The spot density for $\beta-1,3$ glucanase showed remarkable variation in RNA dot blot assay as observed under Alpha Ease FC Imaging software (Alpha Innotech). The control plant showed no spot in RNA dot blot assay, but PGPR inoculated plants showed remarkable differences with and without fungal pathogen infection (Fig. S2 and Table S1). The highest RNA dot blot spot density was observed in plants co-inoculated with $P$. pseudoalcaligenes followed by plants inoculated with both the PGPR in presence of pathogen. The spot was also observed in the plants only inoculated with PGPR in absence of pathogen. Its sequence (Table S2) homology was analyzed by ClustalW software in which it showed close homologies with Oryza sativa mRNA complete cds 
(AF337174.1), Oryza sativa japonica group cDNA clone (AK069096.1) and Oryza sativa (indica cultivar-group) cDNA clone (CT837570.1) with endo-1,3-beta-glucanase.

\section{Molecular modeling of glucanase and docking studies}

The 3D structure of the beta-1,3-glucanase(Oryza sativa indica) has not been solved yet experimentally. Thus, we built a 3D model of the beta-1,3-glucanase protein using homology modelling approach. BLASTp search against PDB resulted in four templates i.e., 1GHS_A:Hordeum vulgare; 2CYG_A: Musa acuminate; 4HPG_A: Hevea brasiliensis; 3UR7_A: Solanum tuberosum (Table S3). Based on the pair-wise sequence alignment between glucanase and the templates using Multalin program protein models were predicted (Fig. S3). After obtaining the final model, overall stereo-chemical qualities of the model were evaluated to ensure its suitability for carrying out the further studies.

Stereo chemical quality of modelled glucanase structure (Fig. S4A, B) was explored using Ramachandran plot. Procheck was employed to validate the rationality of homology model. Ramachandran plot analysis displayed the dihedral angles $\Phi$ against $\Psi$ of amino acid residues in the predicted model (Fig. S4C), where $93.1 \%$ residues were found to be in the most favored region and none of the residues with bad geometry (outlier region of $\Phi$ and $\Psi$ plot) depicting good quality of the model. ERRAT program was used to assess the overall quality factor of the modelled protein by determining the false statistics of bad non-bonded interactions within the structure.

The overall quality factor of the modelled structure was $86 \%$ which indicates that the proposed model is of good quality. Additional analysis of the structure using the Profile-3D score showed comparatively a good score of $98.45 \%$ indicating the accuracy of homology model. Analysis of the predicted model using ProQ server indicated LG-score of "extremely good model" and Max-Sub of "very good model" quality measures. ProSA-Web (Fig.S4D) analysis exhibited the energy profile and Z-score of the model. Z-score for the predicted model was found to be within the range of scores typically found for the native proteins of similar size while the plot of energies of each residue revealed that entire calculated values were negative. Overall, the various model validation servers suggested that the generated model is reliable (Table 1) and thus can be used for docking studies with higher confidence. Furthermore, we made structural imposition of our proposed model with structural templates (1GHS: Hordeum vulgare; 2CYG:Musa acuminata; 4HPG:Hevea brasiliensis; 3UR7: Solanum tuberosum) displayed least RMSD (below $0.8 \AA$ ) indicating the overall good quality of our model structure (Fig. S5).

Molecular docking was performed using AutoDock to understand the mode of interaction of GTP in the ligand binding interface of glucanase. As evidenced from the top ranked conformation (as shown in Fig. 1A, B), the GTP prefers to bind in the cavity formed in glucanase receptor (Table 2). The resulted docking conformation from molecular docking experiment having lowest binding energy of $-4.97 \mathrm{kcal} / \mathrm{mol}$ was selected as the representative structure for protein-ligand interaction studies. Tyr75, Arg256, Gly258, and Ser223 of glucanase formed four crucial hydrogen bonds with the GTP at an atomic distance 3.18, 3.03, 2.24 , and $2.03 \AA$, respectively. While the Val220 of glucanase displayed hydrophobic contacts with GTP (Fig. 1).

\section{MD trajectory analysis}

To understand the dynamic behavior of glucanase-GTP complex, we performed all-atoms MD simulations of complex using GROMACS. To gauge the stability and conformational flexibility of the complex, we computed various properties of the system. The backbone RMSD analysis offers vital information on the stability of protein-ligand complexes and the time when simulation reached equilibrium. RMSD profiles of glucanase-GTP complex displayed
Table 1 Model validation statistics of glucanase subunit of rice using various structural evaluation servers

\begin{tabular}{llc}
\hline Model validation servers & Model quality parameters & Validation scores \\
\hline Procheck (Ramachandran plot) & Most favored regions (\%) & 93.1 \\
& Additional allowed regions (\%) & 6.4 \\
& Generously allowed regions (\%) & 0.5 \\
& Disallowed regions (\%) & 0.0 \\
Verify 3D & Averaged 3D-1D score $\geq 0.2(\%)$ & 98.45 \\
ERRAT & Overall quality (\%) & 86.0 \\
ProSA & Z score & -8.42 \\
ProQ & LG score & 6.33 \\
& Max Sub & 0.61 \\
Prove & Z score mean & -0.06 \\
METAMQAP-II & GDT_TS & 58.69 \\
\hline
\end{tabular}




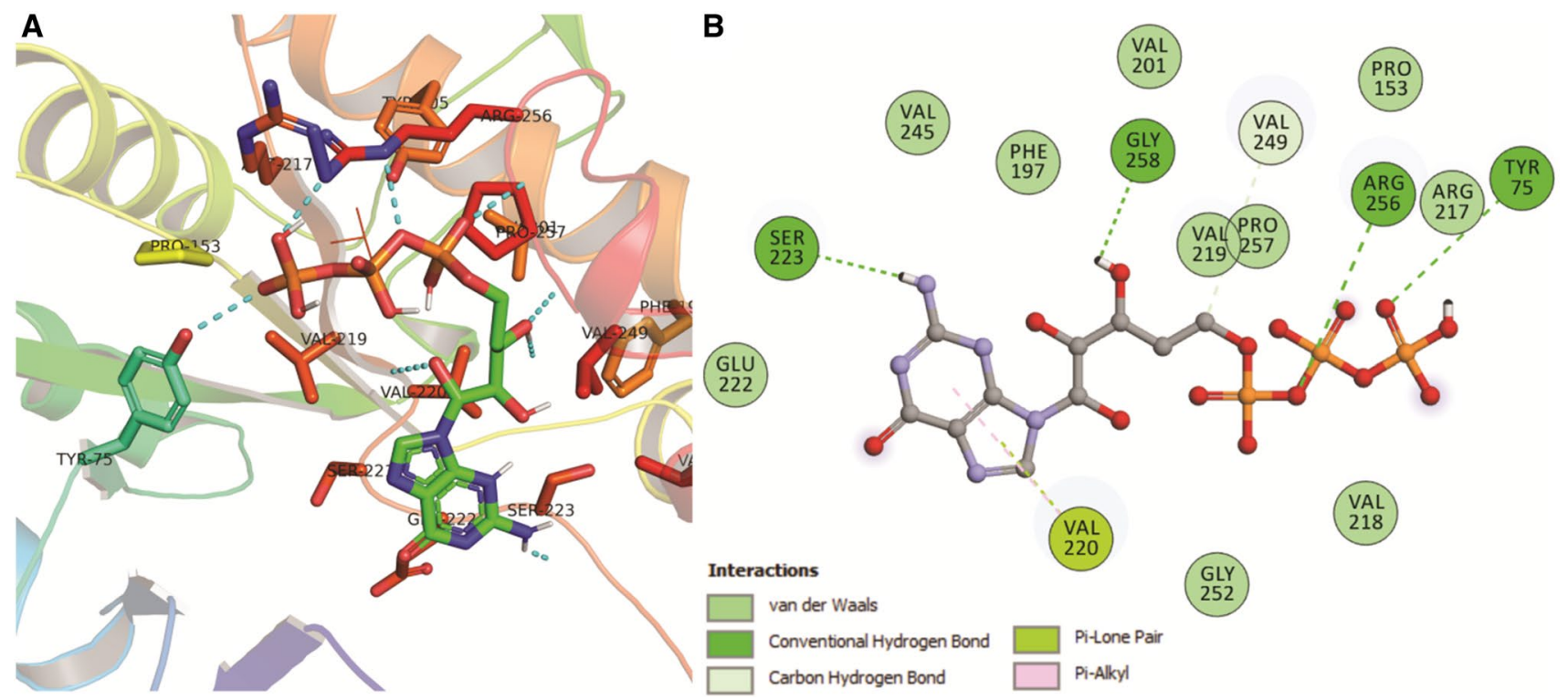

Fig. 1 Inter-molecular contact analysis of GTP with modelled glucanase obtaining after docking using AutoDock. A Interaction of GTP with modelled glucanase using PyMOL where the dotted lines depict hydrogen bonds and the interacting amino acids along with the ligand (stick format). B Non-bonded contact of GTP with glucanase using BIOVIA DSV (hydrogen bond forming amino acids marked in dark green). (Color figure online)
Table 2 Intermolecular contacts of the docked conformation of Glucanase-GTP complex obtained from AutoDock analyzed using BIOVIA DSV

\begin{tabular}{llll}
\hline Interacting pairs & Distance & Type & Category \\
\hline TYR75: OH: GTP: O17 & 3.17837 & Hydrogen bond & Conventional hydrogen bond \\
ARG256:NE: GTP: O8 & 3.03002 & Hydrogen bond & Conventional hydrogen bond \\
GTP:H40: GLY258: OXT & 2.24216 & Hydrogen bond & Conventional hydrogen bond \\
GTP:H44: SER223:O & 2.02791 & Hydrogen bond & Conventional hydrogen bond \\
GTP:C27: VAL249:O & 3.48525 & Hydrogen bond & Carbon hydrogen bond \\
VAL220:O: GTP & 2.77519 & Other & Pi-lone pair \\
GTP: VAL220 & 4.62512 & Hydrophobic & Pi-alkyl \\
GTP: VAL220 & 5.02469 & Hydrophobic & Pi-alkyl \\
\hline
\end{tabular}

least deviation with average RMSD of $\sim 0.32 \mathrm{~nm}$ during the simulation period and by the time of $20 \mathrm{~ns}$ system achieved convergence (Fig. 2A). In addition, the RMSD of ligand (as shown in red line in Fig. 3A) was also found to be stable (below $0.1 \mathrm{~nm}$ ) with minimum deviation. In toto, the docked confirmation remained accurate by manifesting least backbone deviation and stable for $50 \mathrm{~ns}$ timescale.

Radii of gyration determines the robustness of the system; where, gyradius of $\sim 1.85 \mathrm{~nm}$ implies the consistency of size and shape of the system while performing simulation (Fig. 2B).Glucanase-GTP complex flexibility has been evaluated suing C $\alpha$ RMSF analysis (Fig. 2C). As compared to the $\mathrm{N}$-terminal end, the $\mathrm{C}$-terminal end had major fluctuations with higher RMSF values, while the loops also displayed higher peaks indicating flexible nature of the mobile loops during MD.
The intermolecular hydrogen bonds (H-bonds) in a protein-ligand complex plays dynamic role in stability and molecular recognition process. The intermolecular $\mathrm{H}$-bonds were calculated with respect to time during the $50 \mathrm{~ns}$ MD simulation to see the dynamic stability of glucanase-GTP complex (Fig. 2D). We observed an average of $\sim 4.04 \mathrm{H}$-bonds/time frame during the $50 \mathrm{~ns}$ time scale of MD. Snapshots from MD revealed that some of the H-bonds were broken during MD simulation, however, later they well remunerated by new non-bonded contacts. Minute observation of the ligand with respect to the initial starting structure used MD revealed that the GTP prefers to reorient within the ligand binding site during MD but form close tight network of hydrogen bonds and non-bonded contact with glucanase of rice. Clustering analysis through RMSD-based clustering employed in gmx cluster exposed the crucial residues 

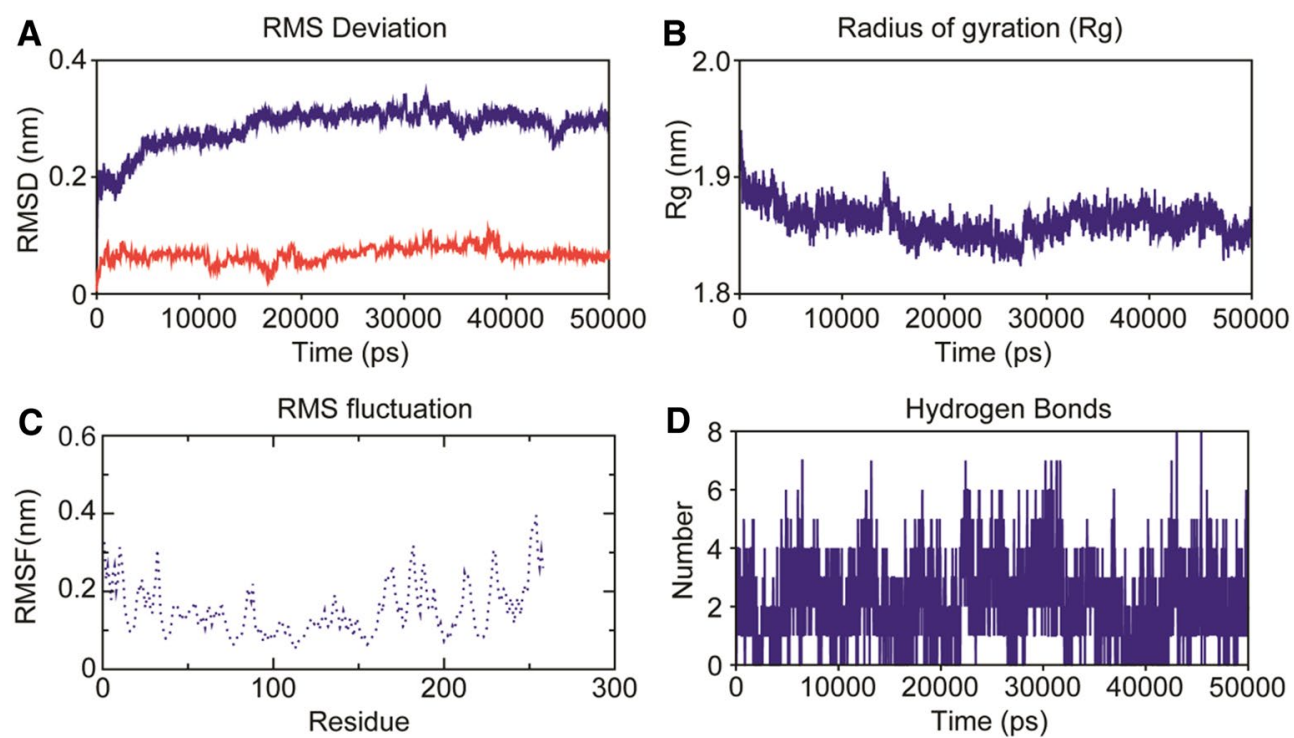

Fig. 2 Dynamics stability of the glucanase-GTP complex system during $50 \mathrm{~ns}$ MD. A Backbone RMSD of the complex and the ligand over the time scale of $50 \mathrm{~ns}$. B Radius of gyration profile indicating

the compactness of the system during $50 \mathrm{~ns}$. C The C- $\alpha$ RMSF profile of the glucanase-GTP complex during the last $30 \mathrm{~ns}$ MD. D Intermolecular H-bond analysis of GTP with glucanase during $50 \mathrm{~ns}$ MD

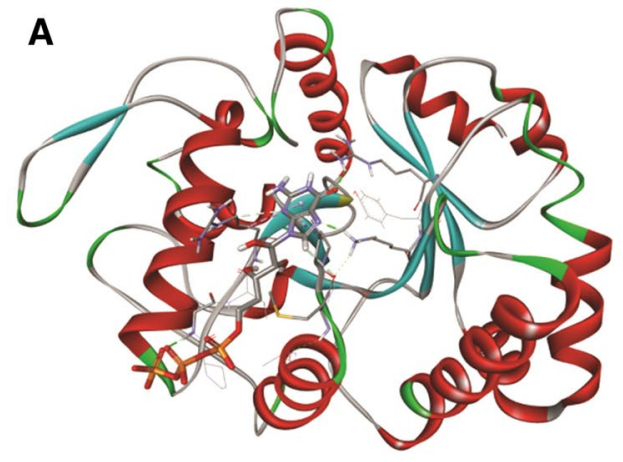

C
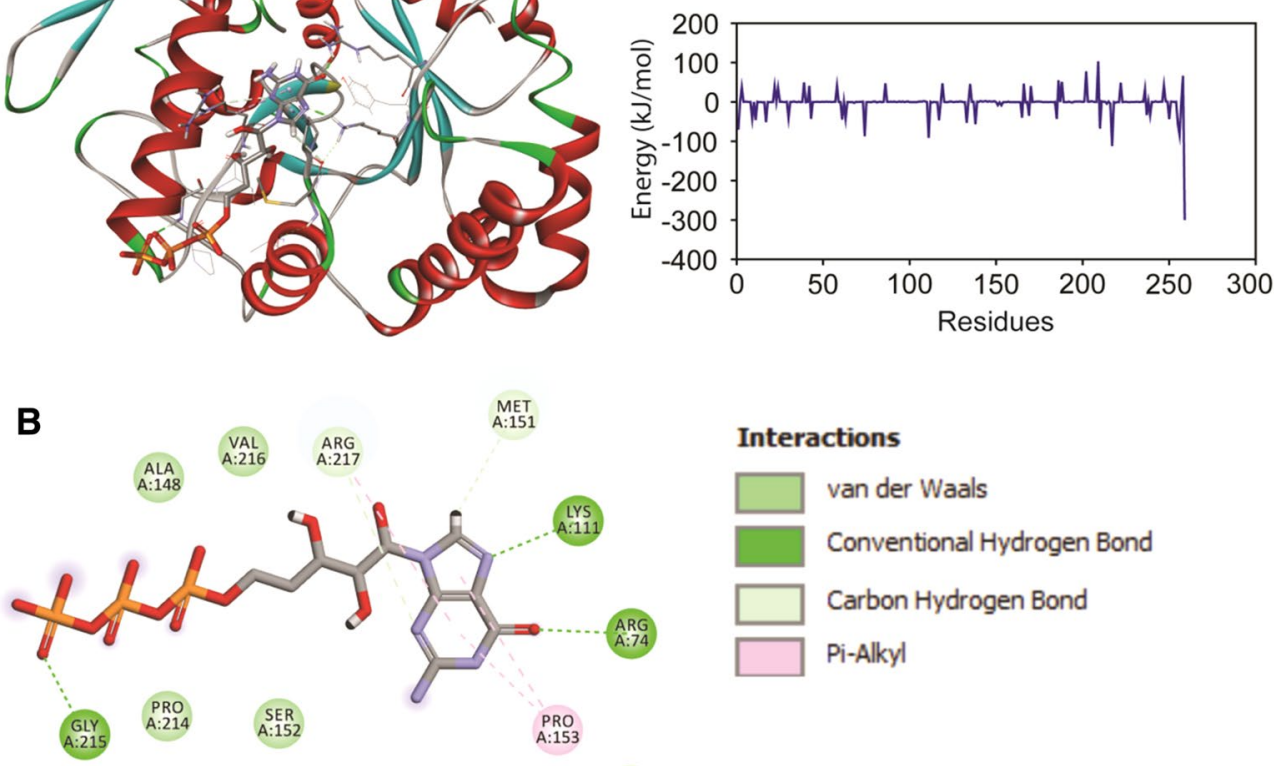

Fig. 3 Inter-molecular contact analysis of the glucanase-GTP complex post MD and the binding free energy decomposition analysis displaying the contribution each residue towards binding free energy. A Snapshot of the Glucanase-GTP complex obtained post MD using

clustering approach. B Inter-molecular contacts of the top ranked cluster displaying the non-bonded contacts. C Free energy decomposition analysis displaying the energetic contribution of each residue towards binding free energy 
of glucanase involved in the important interaction with GTP (Table S4). Arg74, Lys111 and Gly215 formed strong H-bonds while, Pro153and Arg217 formed hydrophobic pialkyl contacts with the ligand GTP (Fig. 3A, B).

The results from PCA have been summarized in Fig. S6. Porcupine plots of top two PCs displayed prominent inward motion in the encircled helical segments of Glucanase. Mostly the distal end at the terminals displayed high degree of in-ward motion. We also computed binding free energy of the glucanase-GTP complexes using snapshots from MD trajectory through MM/PBSA approach implemented in g_mmpbsa tool. A total of 200 snapshots from the last 20 ns of MD were used for computing the binding free energy. Among the energetic terms Van der waals energy, electrostatic energy and SASA energy contributed to the total binding free energy of the system while polar solvation showed positive energy i.e., opposes the binding (Table 3).

To find out the contribution made by each residue towards negative free energy, we also computed the free energy decomposition analysis which revealed a number of amino acids from the binding pocket contributes significantly towards the total binding free energy (Fig. 3C). The contribution made from electrostatic energy signifies the glucanase-GTP complex possess a number of electrostatic contacts as evident from the non-bonded contact analysis. We also extracted the top ranked cluster from MD trajectory to inspect the inter-molecular contacts mediated by glucanase with GTP (table which perfectly fits with the energetic contribution derived MM/PBSA analysis.

\section{Discussion}

Plant-microbe interactions are a common and natural event, which has either beneficial or hostile effect on plant host. Plant also continuously encounter with a wide range of pathogenic microbes having deleterious effect on host plant [28]. A plethora of physical and chemical defense systems have been developed by the plants during the evolutionary process to block the hostile interactions with the different biotic factors. So plants develop a multilayer complex networking

Table 3 Binding free energy of the Glucanase-GTP complex estimated using MM/PBSA approach

\begin{tabular}{ll}
\hline Energetic terms & $\begin{array}{l}\text { Energetic } \\
\text { contribution }(\mathrm{kJ} \\
\left.\mathrm{mol}^{-1}\right)\end{array}$ \\
\hline van der Waal energy & $-109.27 \pm 1.10$ \\
Electrostatic energy & $-748.29 \pm 9.85$ \\
Polar solvation energy & $290.56 \pm 7.79$ \\
SASA energy & $-12.95 \pm 0.17$ \\
Binding energy & $-579.78 \pm 5.42$ \\
\hline
\end{tabular}

pathways to fight with the pathogen, which include the synthesis of PR new proteins, cell lignifications, phytohormone stimulation and induction of defense related gene. Among these chemical defense systems, inhibition of biochemical and enzymatic activities of pathogens or accumulation of lytic secondary metabolites to inhibit the growth or kill the pathogens are predominant mechanisms.

Intensive interactions of microbes with plant roots always take place- as it is a source of diverse and rich microbial foods due to which it acts as active ecological niche [29]. PGPR used to adapt to the specific plant environment in which they colonize and therefore have the higher metabolic potential. Such interaction of plant with PGPR helps in maintaining the plant health by improving the nutrient status, resistant against phyto-pathogens and also to the environmental stresses. The PR protein, $\beta-1,3$ glucanases produced by PGPR in host plant are responsible for hydrolyzing the cell walls of fungus and releasing of $\beta$-1,3-glucan, so directly involved in the protection of plant against the wide range of fungal pathogens [30]. The released $\beta-1,3$-glucan like oligosaccharides can act as an elicitor, which elicit plant defense mechanism. The plant surveillance systems are able to recognize these elicitors, and induce the signaling cascades for the activation of systemic and localized defense responses against the wide range of phytopathogens. $\beta-1,3-$ glucanases is highly effective against fungal pathogen attack as it degrades the fungal cell wall during the pathogen attack.

In this study, we observed that inoculation of paddy with $P$. pseudoalcaligenes and B. pumilus enhanced the plant protection against fungus Magnaportha grisea. There are several reports mentioning that inoculation of plants with the selective PGPR is able to induce protection against a wide range of pathogens. ISR is triggered by PGPR, which results in activation of phytohormones mediated signaling pathways [31], production of defense modulation proteins and induction of pathogenesis-related genes to develop the plant ability to fight against wide range of pathogens attack. Microbes interaction triggers PGPR signaling compounds like cell wall components, chitin oligomers and lipopolysaccharides that elicit ISR in plant [32]. ISR-inducing PGPR have been shown to be effective for the disease control in tomato [12].

A 3D model of the beta-1,3-glucanase (glucanase) protein using homology modelling approach was constructed after selecting the best template that was validated by several servers. Overall, the various model validation servers suggested that the generated model is reliable and thus can be used for docking studies with higher confidence. Molecular docking was performed to understand the mode of interaction of GTP in the ligand binding interface of glucanase. As evidenced from the top ranked conformation, the GTP prefers to bind in the cavity formed in glucanase receptor. A number of hydrogen bonds mediated by Tyr75, Arg256, 
Gly258, and Ser223 form strong interaction with GTP while Val220 alone formed hydrophobic contact. To understand the dynamic behavior of glucanase-GTP complex, we performed all-atoms MD simulations of complex using GROMACS. To gauge the stability and conformational flexibility of the complex, we computed various properties of the system. Overall, the complex system displayed the least backbone deviation, indicating that docked conformation is accurate and remained stable over the $50 \mathrm{~ns}$ timescale.

Radius of gyration assesses the compactness of the system, where a compact gyradius of $\sim 1.85 \mathrm{~nm}$ for the complex indicates the consistent shape and size of the system during simulation. The residue flexibility of glucanase-GTP complex was examined by performing C $\alpha$ RMSF analysis. As compared to the $\mathrm{N}$-terminal end, the $\mathrm{C}$-terminal end had major fluctuation with higher RMSF values. The intermolecular hydrogen bonds (H-bonds) in a protein-ligand complex plays dynamic role in stability and molecular recognition process. The intermolecular $\mathrm{H}$-bonds were calculated with respect to time during the $50 \mathrm{~ns}$ MD simulation to see the dynamic stability of glucanase-GTP complex. We observed an average of $\sim 4.04 \mathrm{H}$-bonds/time frame during the $50 \mathrm{~ns}$ time scale of MD. The residues which formed close contact with the ligand contributed significantly to the overall binding free energy of the complex. Overall, the stability of the complex system and intrinsic dynamics inferred from this study enables us to understand the molecular recognition of GTP with glucanase and advocates to undertake site directed mutagenesis study to confirm the role of ligand binding pocket residues in binding to GTP.

Globally the researchers have been recommending the application of PGPR as plant growth promoting and biocontrol agents, leading to a green and safe sustainable agriculture practices by reducing the various chemical inputs [33, 34]. Usually, applications of compatible microbial consortiums have proven to be better than single inoculant [35]. There are many publications in literature explaining the mechanism behind these actions [31], but none of them have ever proved this phenomenon complementing the insilico and wet lab experiments at the molecular level. Our investigations will fill this gap in knowledge existing in the literature and will work as a backbone for the future bioinformatics-based investigation of mechanism of PGPR action.

This study showed that association of PGPR with plant roots alone is responsible for the induction of plant defense gene expression. Findings of the study indicate that the mixture of both $P$. pseudoalcaligenesand B. pumilus confer better effective resistance by efficient induction of the defense in plant with comparison to the individual isolate. RT-PCR and RNA dot blot analysis both implies that plant defense gene $\beta$-1,3-glucanases is induced at significant level in the plant upon inoculation with PGPR to induce the plant defense. To assess the differential level of mRNA production due to induction by PGPR and after fungal infection in plants, RNA-Dot blot assay was used and results showed that PGPR were able to induce significant level of mRNA in plant to provide the defense against fungal pathogen. However, fungal infection does not show any remarkable variation in spot density of RNA in RNA-Dot blot assay.

So, the finding showed that plant inoculated with mixture of $P$. pseudoalcaligene and B. pumilus confers significant resistance against plant pathogen by inducing changes in the associated gene expression in plant. Moreover, such induction in defense associated gene expression in plant is initiated alone by PGPR prior to the pathogenic interaction. The interaction of plant growth promoting bacteria with plant during the initial stage of development induce multi level change in gene expression cause related change in plant physiology to overcome the adverse effect of stresses has been better analyzed by multi-omics to study the modulatation of cellular mechanisms in plants has been also reported by Meena et al. [36].

Supplementary Information The online version contains supplementary material available at https://doi.org/10.1007/s11033-021-07059-5.

Acknowledgements Authors are thankful to CABin grant (F. no. Agril. Edn.4-1/2013-A\&P), Indian Council of Agricultural Research, Ministry of Agriculture and Farmers' Welfare, Govt. of India. Funding for open access charge: University of Vigo / CISUG.

Author contributions YJ, RBS and SK conceived theme of the study and generated data. YJ collected samples and performed the wet lab experiment. SK, BD and MY did the computational analysis. YJ, SK, BD, SPJK, and JS-G drafted the manuscript. YJ, SK, BD, AC, SPJK, UBS, RBS, RR, MT, UBA and JS-G edited the manuscript. All coauthors read and approved the final manuscript.

Funding Open Access funding provided thanks to the CRUE-CSIC agreement with Springer Nature.

\section{Declarations}

Conflict of interest The authors declare that they have no conflict of interest.

Ethical approval This article does not contain any studies with human participants or animals performed by any of the authors.

Open Access This article is licensed under a Creative Commons Attribution 4.0 International License, which permits use, sharing, adaptation, distribution and reproduction in any medium or format, as long as you give appropriate credit to the original author(s) and the source, provide a link to the Creative Commons licence, and indicate if changes were made. The images or other third party material in this article are included in the article's Creative Commons licence, unless indicated otherwise in a credit line to the material. If material is not included in the article's Creative Commons licence and your intended use is not permitted by statutory regulation or exceeds the permitted use, you will need to obtain permission directly from the copyright holder. To view a copy of this licence, visit http://creativecommons.org/licenses/by/4.0/. 


\section{References}

1. Olanrewaju OS, Glick BR, Babalola OO (2017) Mechanisms of action of plant growth promoting bacteria. World J Microbiol Biotechnol 33(11):197

2. Kumar SPJ, Prasad SR, Banerjee R, Thammineni C (2015) Seed birth to death: dual functions of reactive oxygen species in seed physiology. Ann Bot 116(4):663-668

3. Toruño TY, Stergiopoulos I, Coaker G (2016) Plant-pathogen effectors: Cellular probes interfering with plant defenses in spatial and temporal manners. Annu Rev Phytopathol 4:419-441

4. Kumar SPJ, Chintagunta AD, Reddy YM, Rajjou L, Kumar GV, Agarwal DK, Prasad SR, Simal-Gandara J (2021) Implications of reactive oxygen and nitrogen species in seed physiology for sustainable crop productivity under changing climate conditions. Curr Plant Biol 2021:100197

5. Zandalinas SI, Sales C, Beltrán J, Gómez-Cadenas A, Arbona V (2017) Activation of secondary metabolism in citrus plants is associated to sensitivity to combined drought and high temperatures. Front Plant Sci 7:1954

6. Sarangi S, Mandal C, Dutta S, Mukherjee P, Mondal R, Kumar SPJ, Choudhury PR, Singh VP, Tripathi DK, Mandal AB (2019) Microprojectile based particle bombardment in development of transgenic indica rice involving AmSOD gene to impart tolerance to salinity. Plant Gene 19:100183

7. Kumar A, Kumar SPJ, Chintagunta AD, Agarwal DK, Pal G, Singh AN, Simal-Gandara J (2021) Biocontrol potential of Pseudomonas stutzeri endophyte from Withania somnifera (Ashwagandha) seed extract against pathogenic Fusarium oxysporum and Rhizoctonia solani. Arch Phytopathol Pflanzenschutz 2021:1-18

8. Golshani F, Fakheri BA, Behshad E, Vashvaei RM (2015) PRs proteins and their mechanism in plants. Biol Forum Int $\mathrm{J}$ $7: 477-495$

9. Kumar A, Agarwal DK, Kumar S, Reddy YM, Chintagunta AD, Saritha KV, Pal G, Kumar SPJ (2019) Nutraceuticals derived from seed storage proteins: implications for health wellness. Biocatal Agric Biotechnol 17:710-719

10. Shirinbayan S, Khosravi H, Malakouti JM (2019) Alleviation of drought stress in maize (Zea mays) by inoculation with Azotobacter strains isolated from semi-arid regions. Appl Soil Ecol 133:138-145

11. Egamberdieva D, Wirth S, Jabborova D, Räsänen LA, Liao H (2017) Coordination between Bradyrhizobium and Pseudomonas alleviates salt stress in soybean through altering root system architecture. J Plant Interact 12(1):100-107

12. Suryadi Y, Susilowati DN, Fauziah F (2019) Management of plant diseases by PGPR-mediated induced resistance with special reference to tea and rice crops. Plant Growth Promot Rhizobact Sustain Stress Manage 2019:65-110

13. Jha Y (2019) The importance of zinc-mobilizing rhizosphere bacteria to the enhancement of physiology and growth parameters for paddy under salt-stress conditions. Jordan J Biolo Sci 12(2):167-173

14. Dash PK (2013) High quality RNA isolation from ployphenol-, polysaccharide-and protein-rich tissues of lentil (Lens culinaris). 3Biotech 3(2):109-114

15. Fazil MH, Kumar S, Farmer R, Pandey HP, Singh DV (2012) Binding efficiencies of carbohydrate ligands with different genotypes of cholera toxin B: molecular modeling, dynamics and docking simulation studies. J Mol Model 18(1):1-10

16. Yadav S, Kushwaha HR, Kumar K, Verma PK (2012) Comparative structural modeling of a monothiol GRX from chickpea: Insight in iron-sulfur cluster assembly. Int J Biol Macromol $51: 266-273$
17. Tandon G, Jaiswal S, Iquebal MA, Kumar S, Kaur S, Rai A, Kumar D (2015) Evidence of salicylic acid pathway with EDS1 and PAD4 proteins by molecular dynamics simulation for grape improvement. J Biomol Struct Dyn 33(10):2180-2191

18. Kumar S, Ahmad K, Tandon G, Singh UB, Jha Y, Nagrale DT, Singh MK, Girdhar K, Mondal P (2018) Novel insight into the molecular interaction of catalase and sucrose: a combination of in silico and in planta assays study. Comput Electron Agric 151:258-263

19. Corpet F (1988) Multiple sequence alignment with hierarchical clustering. Nucleic Acids Res 16(22):10881-10890

20. Sali A, Blundell TL (1993) Comparative protein modelling by satisfaction of spatial restraints. J Mol Biol 234(3):779-815

21. Webb B, Sali A (2016) Comparative protein structure modeling using MODELLER. Curr Protoc Bioinformatics 20(54):5.6.1-5.6.37

22. Laskowski RA, Moss DS, Thornton JM (1993) Main-chain bond lengths and bond angles in protein structures. J Mol Biol 231(4):1049-1067

23. Morris GM, Huey R, Lindstrom W, Sanner MF, Belew RK, Goodsell DS, Olson AJ (2009) AutoDock4 and AutoDockTools4: Automated docking with selective receptor flexibility. J Comput Chem 30(16):2785-2791

24. Dehury B, Patra MC, Maharana J, Sahu J, Sen P, Modi MK, Choudhury MD, Barooah M (2014) Structure-based computational study of two disease resistance gene homologues ( $\mathrm{Hml}$ and $\mathrm{Hm} 2$ ) in maize (Zea mays L.) with implications in plantpathogen interactions. PLoS ONE 9(5):e97852

25. Kumar S, Dehury B, Tandon G, Jaiswal S, Iquebal MA, Ahmad K, Nagrale DT, Singh UB, Jha Y, Singh MK, Singh A, Rai A, Paital B, Kumar D (2020) An insight into molecular interaction of PGIP with PG for banana cultivar. Front Biosci 25:335-362

26. Vanommeslaeghe K, MacKerell AD (2012) Automation of the CHARMM General Force Field (CGenFF) I: bond perception and atom typing. J Chem Inf Model 52(12):3144-3154

27. Abraham MJ, Murtola T, Schulz R, Páll S, Smith JC, Hess B, Lindahl E (2015) GROMACS: High performance molecular simulations through multi-level parallelism from laptops to supercomputers. SoftwareX 1:19-25

28. Pereira SIA, Abreu D, Moreira H, Vega A, Castro PML (2020) Plant growth-promoting rhizobacteria (PGPR) improve the growth and nutrient use efficiency in maize (Zea mays L.) under water deficit conditions. Heliyon 6(10):e05106

29. Jha Y, Subramanian RB (2016) Regulation of plant physiology and antioxidant enzymes for alleviating salinity stress by potassium-mobilizing bacteria. In: In: Meena V, Maurya B, Verma J, Meena R (eds) Potassium solubilizing microorganisms for sustainable agriculture. Springer, New Delhi

30. Gowtham HG, Singh BS, Murali M, Shilpa N, Prasad M, Aiyaz M, Amruthesh KN, Niranjana SR (2020) Induction of drought tolerance in tomato upon the application of ACC deaminase producing plant growth promoting rhizobacterium Bacillus subtilis Rhizo SF 48. Microbiol Res 234:126422

31. Backer R, Rokem JS, Ilangumaran G, Lamont J, Praslickova D, Ricci E, Subramanian S, Smith DL (2018) Plant growthpromoting rhizobacteria: Context, mechanisms of action, and roadmap to commercialization of biostimulants for sustainable agriculture. Front Plant Sci 23:1473

32. Jha Y (2019) Endophytic bacteria as a modern tool for sustainable crop management under stress. In: Giri B, Prasad R, Wu QS, Varma A (eds) Biofertilizers for sustainable agriculture and environment. Springer, Cham. https://doi.org/10.1007/ 978-3-030-18933-4_9

33. Chaurasia A, Meena BR, Tripathi AN, Pandey KK, Rai AB, Singh B (2018) Actinomycetes: an unexplored microorganisms 
for plant growth promotion and biocontrol in vegetable crops. World J Microbiol Biotechnol 34(9):132

34. Suárez-Moreno ZR, Vinchira-Villarraga DM, Vergara-Morales DI, Castellanos L, Ramos FA, Guarnaccia C, Degrassi G, Venturi V, Moreno-Sarmiento N (2019) Plant-growth promotion and biocontrol properties of three Streptomyces spp. Isolates to control bacterial rice pathogens. Front Microbiol 25(10):290

35. Bradáčová K, Florea AS, Bar-Tal A, Minz D, Yermiyahu U, Shawahna R, Kraut-Cohen J, Zolti A, Erel R, Dietel K, Weinmann M, Zimmermann B, Berger N, Ludewig U, Neumann G, Poşta G (2019) Microbial consortia versus single-strain inoculants: an advantage in pgpm-assisted tomato production? Agronomy 9(2):105. https://doi.org/10.3390/agronomy9020105
36. Meena KK, Sorty AM, Bitla UM, Choudhary K, Gupta P, Pareek A, Singh DP, Prabha R, Sahu PK, Gupta VK, Singh HB, Krishanani KK, Minhas PS (2017) Abiotic Stress Responses and Microbe-Mediated Mitigation in Plants: The Omics Strategies. Front Plant Sci 8:172

Publisher's Note Springer Nature remains neutral with regard to jurisdictional claims in published maps and institutional affiliations.

\section{Authors and Affiliations}

\section{Yachana Jha ${ }^{1,9} \cdot$ Budheswar Dehury $^{2}$ - S. P. Jeevan Kumar ${ }^{3}$. Anurag Chaurasia ${ }^{4}$. Udai B. Singh ${ }^{5}$. Manoj Kumar Yadav $^{6}$. U. B. Angadi ${ }^{10} \cdot$ Rajiv Ranjan $^{7} \cdot$ Minaketan Tripathy $^{8} \cdot$ R. B. Subramanian ${ }^{9} \cdot$ Sunil Kumar $^{5,10}$. Jesus Simal-Gandara ${ }^{11}$}

Sunil Kumar

skybiotech@gmail.com

$\square$ Jesus Simal-Gandara jsimal@uvigo.es

$1 \quad$ N. V. Patel College of Pure and Applied Sciences, S.P. University, Anand 388315, India

2 ICMR-Regional Medical Research Centre, Bhubaneswar 751023, India

3 ICAR-Directorate of Floricultural Research, Pune 411036, Maharashtra, India

4 ICAR- Indian Institute of Vegetable Research, Varanasi 221305, India

5 ICAR-National Bureau of Agriculturally Important Microorganisms, Mau 275103, Uttar Pradesh, India

6 SRM University, Sonepat 131029, Haryana, India
7 Dayalbagh Educational Institute, Agra 282005, Uttar Pradesh, India

8 Adichunchunagiri University, Javarana Hally 571448, Karnataka, India

9 BRD School of Bioscience, Sardar Patel University, Anand 388120, Gujarat, India

10 ICAR-Indian Agricultural Statistics Research Institute, New Delhi 110012, India

11 Nutrition and Bromatology Group, Department of Analytical and Food Chemistry, Faculty of Science, Universidade de Vigo, E32004 Ourense, Spain 